\title{
Effects of Two Weeks of Cerebellar Theta Burst Stimulation in Cervical Dystonia Patients
}

\author{
Giacomo Koch ${ }^{\mathrm{a}, \mathrm{b}, *}$, Paolo Porcacchia ${ }^{\mathrm{c}}$, Viviana Ponzo ${ }^{\mathrm{a}}$, Fatima Carrillo ${ }^{\mathrm{c}}$, María Teresa Cáceres-Redondo ${ }^{\mathrm{c}}$, \\ Livia Brusa $^{\mathrm{d}}$, Maria Teresa Desiato ${ }^{\mathrm{d}}$, Flavio Arciprete ${ }^{\mathrm{d}}$, Francesco Di Lorenzo ${ }^{\mathrm{a}}$, Antonio Pisani ${ }^{\mathrm{b}}$, \\ Carlo Caltagirone ${ }^{\mathrm{a}, \mathrm{b}}$, Francisco J. Palomar ${ }^{\mathrm{c}, \mathrm{e}}$, Pablo Mir $^{\mathrm{c}, \mathrm{e}, * *}$ \\ a Non-invasive Brain Stimulation Unit, Santa Lucia Foundation IRCCS, Via Ardeatina 306, 00179 Rome, Italy \\ ${ }^{\mathrm{b}}$ University of Rome Tor Vergata, Viale Oxford 81, 00133 Rome, Italy \\ ${ }^{\mathrm{c}}$ Unidad de Trastornos del Movimiento, Servicio de Neurología y Neurofisiología Clínica, Instituto de Biomedicina de Sevilla, \\ Hospital Universitario Virgen del Rocío/CSIC/Universidad de Sevilla, Seville, Spain \\ ${ }^{\mathrm{d}}$ UOC Neurologia, Ospedale S. Eugenio, P.le dell'Umanesimo 10, 00144 Rome, Italy

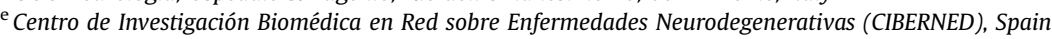

\section{A R T I C L E I N F O}

\section{Article history:}

Received 12 December 2013

Received in revised form

27 April 2014

Accepted 1 May 2014

Available online 29 May 2014

\section{Keywords:}

Cerebellar-thalamo-cortical circuits

Cervical dystonia

cerebellar theta burst stimulation

Paired associative stimulation

\begin{abstract}
A B S T R A C T
Dystonia is generally regarded as a disorder of the basal ganglia and their efferent connections to the thalamus and brainstem, but an important role of cerebellar-thalamo-cortical (CTC) circuits in the pathophysiology of dystonia has been invoked. Here in a sham controlled trial, we tested the effects of two-weeks of cerebellar continuous theta burst stimulation (cTBS) in a sample of cervical dystonia (CD) patients. Clinical evaluations were performed by administering the Toronto Western Spasmodic Torticollis Rating Scale (TWSTRS) and the Burke-Fahn-Marsden Dystonia Rating Scale (BFMDRS). We used TMS to measure the inhibitory connectivity between the cerebellum and the contralateral motor cortex (cerebellar brain inhibition [CBI]), and the excitability of the contralateral primary motor cortex assessing intracortical inhibition (SICI), intracortical facilitation (ICF) and cortical silent period (CSP). Paired associative stimulation (PAS) was tested to evaluate the level and the topographical specificity of cortical plasticity, which is abnormally enhanced and non-focal in CD patients. Two weeks of cerebellar stimulation resulted in a small but significant clinical improvement as measured by the TWSTRS of approximately $15 \%$. Cerebellar stimulation modified the CBI circuits and reduced the heterotopic PAS potentiation, leading to a normal pattern of topographic specific induced plasticity. These data provide novel evidence CTC circuits could be a potential target to partially control some dystonic symptoms in patients with cervical dystonia.
\end{abstract}

C) 2014 Elsevier Inc. All rights reserved.
This work was supported by a generous grant of the Jacques and Gloria Gossweiler Foundation (JGGF) to GK and PM, and by grants of the Italian Ministry of Health to GK (GR-2009-1591859). This work was also supported by grants from the Ministerio de Economía y Competitividad de España [SAF2007-60700]; the Instituto de Salud Carlos III [PI10/01674, CP08/00174], the Consejería de Economía, Innovación, Ciencia y Empleo de la Junta de Andalucía [CVI-02526, CTS-7685], the Consejería de Salud y Bienestar Social de la Junta de Andalucía [PI-0377/2007, PI-0741/ 2010, PI-0437-2012], the SociedadAndaluza de Neurología and the Fundación Alicia Koplowitz. The authors report no disclosures.

* Corresponding author. Non invasive Brain Stimulation Unit, Laboratorio di Neurologia Clinica e Comportamentale, IRCCS Fondazione S. Lucia, Via Ardeatina, 306, 00179 Rome, Italy. Tel.: +39 0651501181.

** Corresponding author. Unidad de Trastornos del Movimiento, Servicio de Neurología y Neurofisiología Clínica, Hospital Universitario Virgen del Rocío, Avda. Manuel Siurot s/n, Sevilla 41013, Spain.

E-mail addresses: g.koch@hsantalucia.it, giakoch@gmail.com (G. Koch),pmir@ us.es (P. Mir).

\section{Introduction}

Dystonia is a movement disorder characterized by excessive involuntary muscle contraction. It is generally considered as a disorder of the basal ganglia and their efferent connections to the thalamus and brainstem, so that the direct pathway is relatively overactive [1]. This imbalance should lead to excessive movement and, in particular, a loss of inhibition at different levels of the nervous system. An interrelated feature of the loss of inhibition is the altered cortical plasticity. Animal models of dystonia have revealed altered patterns of cortical reorganization in the primary somatosensory cortex [2] and abnormal synaptic plasticity at corticostriatal synapses [3]. Subsequent support for abnormalities in cortical plasticity has derived from studies of primary dystonia patients, using indirect measures of long-term potentiation (LTP) 
and depression (LTD), obtained by using techniques such as paired associative stimulation (PAS). PAS combines pairs of peripheral electrical pulses delivered over a nerve (the median nerve, in this case) with magnetic pulses (TMS) delivered over the primary motor cortex (M1). Repeating the pairing of stimuli over an extended period may induce a long-lasting increase or decrease of the excitability of M1 in manner that depends on the interstimulus interval (ISI) [4]. These studies have assessed PAS in focal dystonia, finding enhanced effects consistent with increased plasticity that is evident for the facilitatory PAS with inter-stimulus interval of 20-25 ms [5,6]. Moreover, a key feature of PAS is its topographically organization. Changes in excitability are restricted to the cortical representations of muscles innervated by the peripheral nerve that was stimulated electrically. This topographic specificity of plasticity may be related to the functional somatotopical mapping of somatosensory and motor areas and is markedly reduced in dystonic patients $[5,6]$.

Recent evidence suggested that cerebellar circuits may play a prominent role in the pathophysiology of dystonia [7,8]. For instance, patients affected by primary dystonia have structural, metabolic and functional changes of cerebellar circuits, and MRI investigations revealed that patients with writer's cramp show a gray matter decrease in cerebellum [9]. Indeed, changes in microstructural imaging and metabolic activity of cerebellarthalamo-cortical (CTC) pathways have been observed in primary torsion dystonia [10] and in hereditary dystonia [11]. Using diffusion tensor imaging and probabilistic tractography to identify the specific circuit abnormalities underlying clinical penetrance in carriers of genetic mutations for dystonia, Argyelan and colleagues [12] revealed that the integrity of CTC fiber tracts is reduced in both manifesting and clinically non-manifesting dystonia mutation carriers. Reductions in CTC connectivity correlated with increased motor activation responses, consistently with loss of inhibition at the cortical level. The role of the cerebellum in the pathophysiology of dystonia has also been recognized in cases in which dystonia occurs following a brain lesion (secondary dystonia) [13]. Indeed, dystonia can be seen in cerebellar disorders such as Friederich's ataxia [13] or after cerebellar stroke [14]. In animal models of tottering mice, surgical removal of the cerebellum eliminates the dystonic attacks [15] and there is also evidence for abnormally increased cerebellar activity [16]. Pathological evidence provided strong support for the involvement of the cerebellum in a large sample of patients with cervical dystonia (CD). The $C D$ brains appeared to have a relatively large number of Marinesco bodies in the substantia nigra and showed reduced cerebellar Purkinje neurons, along with related findings of increased torpedo bodies and focal regions of gliosis. Notably, extensive stains failed to reveal any consistent defects in the basal ganglia and in the cerebral cortex [17]. Taken together, these data support the hypothesis that the CTC are involved in the pathogenesis of dystonia [18].

In humans, the functions of the CTC pathway can be studied non-invasively using TMS. It has been proposed that cerebellar TMS activates the Purkinje cells of the posterior cerebellum (lobules VIII and Crus II); such activation results in an inhibition of the dentate nucleus, which is known to exert a background tonic facilitatory drive onto the contralateral motor primary cortex (M1) through synaptic relay in the ventral lateral thalamus $[19,20]$. This in turn leads to an inhibition of the contralateral M1, due to a reduction in the dentate-thalamo-cortical facilitatory drive [21-23]. This form of cortical inhibition has been called cerebellar-brain inhibition (CBI) [21]. Recently, considering previous animal studies showing the existence of both LTP and LTD mechanisms in the cerebellum [24-26], we and others applied over the lateral cerebellum [27,28] different protocols of theta burst stimulation (TBS), a novel form of
rTMS that is capable to induce LTP- and LTD-like effects of the stimulated portion of the human cortex [29]. We found that cerebellar TBS induces bi-directional and long-lasting changes in the excitability of the CTC circuits, possibly activating different mechanisms of synaptic plasticity [27,30]. In particular there was a strong bidirectional modulation of the intracortical inhibitory circuits of the contralateral motor cortex. Further investigations demonstrated that TBS of the cerebellum changes also the CBI interactions [28]. Moreover, it was recently shown that cerebellar TBS modulate the excitability of the posterior cerebellar cortex and condition an ongoing M1 plasticity, changing the PAS induced M1 plasticity [31]. These results gave way to the prospect of modulating the excitability of CTC circuits in vivo, with clear implications to study the physiology of cerebellar plasticity and with possible translational approaches to treat some movements disorders, as recently demonstrated in the case of Parkinson's disease (PD) with levodopa induced dyskinesias [32,33].

On the basis of this background, here we reasoned that cerebellar TBS, by reducing the increased activity of the cerebellum [13] could be able to modulate the functioning of the CTC pathways and eventually lead to a significant clinical improvement in a sample of CD patients.

\section{Materials and methods}

\section{Patients}

Twenty patients affected by primary cervical dystonia as confirmed by clinical examination were enrolled in this doubleblind dual-center placebo-controlled randomized trial since May 2010 until December 2012 (Table 1). All subjects were righthanded, according to the Edinburgh inventory. They all underwent a standard clinical neurologic examination and were recorded during the assessment. The assessment in each video-recorded session consisted of a complete Toronto Western Spasmodic Torticollis Rating Scale (TWSTRS) [34] and the Burke-Fahn-Marsden Dystonia Rating Scale (BFMDRS) [35]. All patients gave informed consent for participation in the study. Experimental procedures were approved by both local ethics committees and conducted in accordance with the Declaration of Helsinki. As a control group for comparison of baseline neurophysiological examinations we studied 10 age matched healthy subject $(7 \mathrm{M}, 3 \mathrm{~F}$; mean age: $51.2 \pm 12.2$ )

\section{Experimental design}

Patients underwent a two-week course of bilateral cerebellar cTBS to evaluate the hypothesis that the long-term modulation of cerebello-cortical interactions could induce some clinical and neurophysiological changes in patients with cervical dystonia. Clinical rating of dystonia was assessed with the TWSTRS and the BFMDRS. These scales were performed by blinded raters during the week before the starting of the first two-week bilateral cerebellar cTBS (pre-cTBS), the Monday following the 2 weeks of stimulation (post-cTBS), 2 weeks and 4 weeks after the end of the stimulation period. Testing sessions required approximately $1 \mathrm{~h}$ for patient. Two experts in the field of movement disorders rated videotapes independently to provide scores. Both video raters were blinded to rTMS condition, and the scores were generated after a consensus was reached comparing the individual scores. The Friday before and the Monday after the cTBS sessions, patients were evaluated with standard TMS methods to explore the functional connectivity between the cerebellar hemisphere and the contralateral M1 (CBI) [21], to measure the excitability of the contralateral primary motor cortex assessing intracortical inhibition (SICI), intracortical 
Table 1

Clinical characteristics of dystonic patients enrolled in the study.

\begin{tabular}{|c|c|c|c|c|c|c|c|}
\hline Patient & $\begin{array}{l}\text { Age } \\
\text { (years) }\end{array}$ & Gender & $\begin{array}{l}\text { Duration of } \\
\text { disease (years) }\end{array}$ & $\begin{array}{l}\text { Predominant } \\
\text { dystonic pattern }\end{array}$ & Additional symptoms & $\begin{array}{l}\text { Time from } \\
\text { botulinum toxin }\end{array}$ & Pharmacological treatment \\
\hline 1 & 66 & Female & 4 & Leftward torticollis & None & 2 months & Tetrabenazine \\
\hline 2 & 63 & Female & 4 & Leftward torticollis & None & 3 months & Tetrabenazine, trihexyphenidyl \\
\hline 3 & 53 & Female & 13 & Leftward torticollis & None & 3 months & - \\
\hline 4 & 54 & Female & 40 & Right torticollis & None & 2 months & - \\
\hline 5 & 33 & Male & 6 & Leftward torticollis & None & 2 years & - \\
\hline 6 & 66 & Female & 15 & Lateral torticollis & Blepharospasm, spasmodic dystonia & 3 months & - \\
\hline 7 & 54 & Female & 27 & Right segmental dystonia & None & 2 months & Lorazepam \\
\hline 8 & 53 & Female & 1 & Leftward torticollis & None & 3 months & - \\
\hline 9 & 63 & Female & 4 & Leftward torticollis & None & 3 months & Tetrabenazine, trihexyphenidyl \\
\hline 10 & 33 & Male & 6 & Leftward torticollis & None & 2 years & - \\
\hline 11 & 59 & Female & 13 & Lateral torticollis & Blepharospasm & 2 months & Venlafaxine \\
\hline 12 & 39 & Female & 13 & Lateral torticollis & Hands dystonia & - & Clonacepam, trihexyphenidyl \\
\hline 13 & 61 & Female & 36 & Right torticollis & Axial dystonia & 3 months & Trihexyphenidyl \\
\hline 14 & 54 & Female & 5 & Lateral torticollis & None & 11 months & - \\
\hline 15 & 64 & Female & 6 & Right torticollis & None & 3 months & - \\
\hline 16 & 34 & Female & 14 & Right torticollis & None & 14 months & - \\
\hline 17 & 45 & Female & 4 & Lateral torticollis & Axial dystonia & 2 months & Clonacepam, trihexyphenidyl \\
\hline 18 & 74 & Female & 7 & Right torticollis & Blepharospasm & 4 months & Clonacepam, tetrabenazine \\
\hline 19 & 40 & Male & 3 & Lateral torticollis & None & 4 months & - \\
\hline 20 & 73 & Male & 60 & Right torticollis & Generalized dystonia & 11 months & Clonacepam, trihexyphenidyl \\
\hline
\end{tabular}

facilitation (ICF) [36] and cortical silent period (CSP), and to assess cortical plasticity involving sensory processing using the PAS protocol [4]. Patients were randomly assigned to real or sham bilateral cerebellum cTBS: ten were assigned to real cTBS and, and the other ten to sham CTBS. CD patients with left and right torticollis were equally distributed in the Sham and Real groups.

Two patients (one in each group) refused to perform all the TBS sessions and were discarded from the study. Patients were unaware of their group assignment. There were 10 days of bilateral cerebellar cTBS stimulation (5 days per week, Monday to Friday), performed at the same hour in the morning for each patient. Two trains of cTBS were applied over the left and the right lateral cerebellum with a pause of 2 min between the 2 trains. The order of stimulation was pseudo-randomized in each subject in every session.

\section{Cerebellar theta burst stimulation}

A MagStim Super Rapid magnetic stimulator (Magstim Company, Whitland, Wales, UK), connected with a figure-of-eight coil with a diameter of $70 \mathrm{~mm}$ was used to deliver cTBS. Three-pulse bursts at $50 \mathrm{~Hz}$ repeated every $200 \mathrm{~ms}$ for $40 \mathrm{~s}$ (600 pulses) were delivered over the lateral cerebellum at $80 \%$ active motor threshold (AMT) $[36,37]$ of the ipsilateral M1 [29,27]. The AMT was determined in the first dorsal interosseous muscle (FDI) as the minimum stimulation intensity that evoked a clearly distinguishable MEP from the background EMG with a tonic muscular contraction of $10 \%$ of maximal voluntary contraction (MVC). cTBS was applied over the lateral cerebellum using the same scalp coordinates $(1 \mathrm{~cm}$ inferior and $3 \mathrm{~cm}$ left/right to the inion) adopted in previous MRI studies showing that this site targets the posterior and superior lobules of the lateral cerebellum [39]. Although cerebellar stimulation has been originally performed with a double cone coil [21] we used the figure-of-eight coil because this approach has been adopted in previous investigations in which cerebellar rTMS was shown to be effective in modulating the excitability of the contralateral motor cortex $[27,31-33,40]$. The coil was positioned tangentially to the scalp, with the handle pointing upwards [27,32]. Sham stimulation was delivered with the coil angled at $90^{\circ}$, with only the edge of the coil resting on the scalp. Stimulus intensity, expressed as a percentage of the maximum stimulator output, was set only at $40 \%$ AMT for the FDI [32].

\section{EMG recordings}

MEPs, elicited by TMS over the left M1, were recorded from the right FDI and abductor pollicis brevis (APB) muscles, using two $\mathrm{Ag}-\mathrm{AgCl}$ surface cup electrodes ( $9 \mathrm{~mm}$ diameter). Responses were amplified with a Digitimer D360 amplifier (Digitimer Ltd., Welwyn Garden City, Hertfordshire, UK) through a band-pass filter set at $30 \mathrm{~Hz}$ (low-pass) and $1 \mathrm{kHz}$ (high-pass) and then recorded by a computer using SIGNAL software for an off-line analysis. The electrophysiological parameters were measured only on the dominant side, given that in dystonic patients the abnormalities of cortical excitability are known to be widespread even to the nonaffected side [6].

\section{CBI protocol}

TMS was performed with two MagStim 200 (Magstim Co., Whitland, Dyfed, UK) connected to two standard figure-of-eight flat coils (70 mm diameter). One coil stimulated the left M1 and MEPs were recorded from the right FDI. The direction of the induced current was from posterior to anterior and was optimal to activate the motor cortex trans-synaptically. We set the intensity for the motor cortical test stimulus (TS) at the intensity that produced MEP of $1 \mathrm{mV}$, with the muscles relaxed. The second coil was used to deliver the conditioning stimuli and it was placed over the right cerebellar hemisphere [21]. For cerebellar stimulation the handle was positioned upwards with the coil placed tangentially to the skull. It was applied over the right lateral cerebellum using the same scalp co-ordinates $(1 \mathrm{~cm}$ inferior and $3 \mathrm{~cm}$ left/right to the inion) [39]. The cerebellar conditioning stimulus intensity was set at $90 \%$ of resting motor threshold (RMT) obtained in the ipsilateral motor cortex $[40,41]$. CS preceded the TS by different interstimulus intervals (ISIs) ranging from 3 to $10 \mathrm{~ms}(3,5,10 \mathrm{~ms})$. There were four conditions, corresponding to the three different ISIs and the TS alone. Ten responses were collected for each different ISI condition and 20 responses were collected with TS alone.

\section{SICI/ICF protocol}

Paired TMS of the left M1 was performed with a 7-cm figure-ofeight coil and 2 Magstim 200 stimulators connected via a Bistim module. The magnetic stimuli had a nearly monophasic pulse 
configuration. The coil was placed at the optimal position for eliciting motor evoked potentials (MEPs) from the right contralateral FDI. SICI was tested using paired TMS with CS preceding a TS by $1-15 \mathrm{~ms}(1,2,3,5,7,10,15 \mathrm{~ms})$ [38]. CS was set at $80 \%$ of AMT while the intensity of TS was adjusted to evoke an MEP of approximately $1 \mathrm{mV}$ peak to peak in the relaxed FDI. There were eight conditions, corresponding to the seven different ISI and the TS alone. Ten responses were collected for each different ISI condition and 20 responses were collected with TS alone.

\section{Cortical silent period (CSP)}

CSP was defined as from the onset of MEP to the visible return of interrupted EMG activity, at a display gain of $500 \mu \mathrm{V} /$ div. MEPs were recorded using surface electrodes from the right FDI muscles after TMS via a figure-of-eight coil (mean diameter of $70 \mathrm{~mm}$ ) connected to a Magstim 200 stimulator (Magstim Company, Whitland, Dyfed, UK). The coil was placed tangentially to the scalp, with the handle pointing backward for FDI. The scalp stimulation was delivered on the site where the largest MEPs were obtained for each FDI. In order to measure the CSP, ten pulses were delivered at $130 \%$ of RMT while the subject maintained constant FDI tonic activity at 50\% of MVC [42].

\section{PAS protocol}

PAS consisted of 225 electrical stimuli applied to the right median nerve at the wrist, paired with focal TMS of the left hand area (M1) at the hot spot optimal for generating MEPs in the right abductor pollicis brevis (APB) muscle. The rate of paired stimulation was $0.25 \mathrm{~Hz}$ applied over a period of $15 \mathrm{~min}$ [43]. Electrical stimulation was delivered through a bipolar electrode (cathode proximal), using constant current square wave pulses (duration, $1 \mathrm{~ms}$ ) at an intensity just above motor threshold to elicit a small $(<200 \mu \mathrm{V})$ M wave [43]. TMS was delivered at ISI $=25 \mathrm{~ms}$ through a focal figure-eight coil (diameter $70 \mathrm{~mm}$ ) connected to a Magstim 200 magnetic stimulator with a near monophasic current waveform (The Magstim Company). The coil was held tangential to the scalp with the handle pointing backward and $45^{\circ}$ away from the midline. MEP amplitudes were measured in the right APB and FDI muscles to assess PAS-induced changes in corticospinal excitability of the hand area of the left M1. The APB was selected as a target muscle because is innervated by the median nerve. The FDI was selected as a control muscle because is innervated by the ulnar nerve [4]. MEPs were elicited in the relaxed APB and FDI muscles of the right hand by TMS applied to the hot spots of the left M1 hand area for each muscle. The excitability of APB and FDI representation was measured for each hot spot independently. TMS intensity was adjusted to elicit MEP $1 \mathrm{mV}$. In both muscles 20 MEPs were recorded before and immediately after PAS. The order of recordings was pseudo-randomized for the presentation of muscle across subjects.

\section{Data analysis}

Non parametric Friedman ANOVAs with Wilcoxon tests were performed between the values of TWSTRS and those of BFMDRS obtained before and 2, 4, and 6 weeks after cTBS in each group. Repeated measures ANOVAs were performed for baseline SICI, SP, PAS and CBI on mean percentage of change in respect to TS with GROUP (cTBS vs. sham vs. HS) and ISI as main factors. For testing the effects on TBS in CD patients other repeated measures ANOVAs were performed for each TMS protocol with GROUP (cTBS vs. sham) as between subjects main factor and with TIME (pre vs. post) and ISI as within subjects' main factors. When a significant main effect was reached, post hoc $t$-tests were employed to characterize the different effects of the specific ISIs. For all statistical analyses, a $P$ value of $<0.05$ was considered to be significant. Mauchley's test examined for sphericity. The Greenhouse-Geisser correction was used for non-spherical data.

\section{Results}

The procedure was well tolerated by all subjects. The mean patients' and healthy subjects' AMT and RMT taken with the MagStim Super Rapid magnetic stimulator did not differ across groups and did not vary before and after the cTBS sessions (Table 2).

We found that two weeks of cerebellar stimulation resulted in a clinical improvement as measured by the TWSTRS only in the cTBS $\left(X^{2}(N=9 ; \mathrm{df}=3)=10.34 ; P=0.01\right)$ but not in the sham group $\left(X^{2}\right.$ $(N=9 ; \mathrm{df}=3)=5.2 ; P=0.12)$. Notably, Mann-Whitney U test did not show any significant difference among the baseline scores in the two groups. Wilcoxon post hoc test showed that in the cTBS group TWRTS were reduced in comparison with baseline after the end of the period of stimulation at the first evaluation $(33.6 \pm 4.2 \mathrm{vs}$. $38.8 \pm 4.1 ; P=0.008$ ), but not at later follow-up. The analysis performed on the global BFMDRS failed to show any significant change, although there was a trend for the cTBS group $\left(X^{2}(N=9\right.$; $\mathrm{df}=3)=6.52 ; P=0.08)$ but not for the sham group $\left(X^{2}(N=9\right.$; $\mathrm{df}=3)=1.69 ; P=0.63)($ Fig. 1$)$.

ANOVA performed on baseline SICI/ICF measures revealed a significant main effect of ISI $(F(6,138)=10.62 ; P=0.00001)$ but not of GROUP $(F(2,23)=0.07 ; P=0.92)$ and GROUP $\times$ ISI $(F(12,138)=0.94 ; P=0.5)$ interaction (Fig. $2 \mathrm{~A})$. ANOVA performed on baseline CSP measures did not show any effect for GROUP main factor $(F(2,25)=0.63 ; P=0.53)$ (Fig. $2 \mathrm{~B})$. When comparing baseline $C B I$ values in $C D$ patients and in healthy subjects repeated measures ANOVA showed an effect of ISI $(F(2,42)=5.02 ; P=0.011)$ but not of GROUP $(F(2,21)=2.60 ; P=0.15)$ nor of GROUP $\times$ ISI interaction $(F(4,42)=0.80 ; P=0.53$ ) (Fig. 2C). Therefore the baseline values of SICI/ICF, CSP and CBI did not differ between CD patients and healthy controls. This finding is consistent with the majority of the previous studies, although some reported decreased inhibition of SICI and CSP [1].

PAS protocol showed that at baseline as expected patients with $\mathrm{CD}$ in both groups showed the described phenomenon of increased plasticity with loss of spatial specificity, consistently with loss of

Table 2

Neurophysiological parameters in the TMS experiments.

\begin{tabular}{|c|c|c|c|c|c|c|c|c|c|c|}
\hline Groups & Age & Cases & Time & FDI $(\mathrm{mV})$ & $\mathrm{ABP}(\mathrm{mV})$ & RMT & $90 \%$ RMT & 70\% RMT & AMT & $80 \%$ AMT \\
\hline \multirow[t]{2}{*}{ cTBS } & $55.2 \pm 13.0$ & 9 & PRE & $52.0 \pm 13.5$ & $52.0 \pm 13.2$ & $42.2 \pm 10.2$ & $38.0 \pm 9.2$ & $29.5 \pm 7.1$ & $47.5 \pm 7.4$ & $38.0 \pm 5.9$ \\
\hline & & & POST & $52.5 \pm 13.2$ & $52.0 \pm 13.2$ & $44.0 \pm 11.4$ & $39.6 \pm 10.3$ & $30.8 \pm 8.0$ & $43.2 \pm 7.8$ & $34.8 \pm 6.4$ \\
\hline \multirow[t]{2}{*}{ SHAM } & $52.6 \pm 11.8$ & 9 & PRE & $53.0 \pm 13.3$ & $56.2 \pm 16.2$ & $47.0 \pm 7.7$ & $42.3 \pm 6.9$ & $32.9 \pm 5.4$ & $46.2 \pm 5.4$ & $37.0 \pm 4.4$ \\
\hline & & & POST & $54.6 \pm 14.6$ & $56.0 \pm 16.0$ & $44.6 \pm 11.1$ & $40.1 \pm 10.0$ & $35.9 \pm 16.7$ & $38.4 \pm 7.7$ & $30.7 \pm 6.2$ \\
\hline HS & $51.2 \pm 12.2$ & 10 & PRE & $50.4 \pm 9.7$ & $53.0 \pm 8.2$ & $40.3 \pm 9.1$ & $36.2 \pm 7.1$ & $28.2 \pm 8.2$ & $39.4 \pm 4.3$ & $31.5 \pm 4.7$ \\
\hline
\end{tabular}

$\mathrm{PRE}=$ pre cTBS; POST = post cTBS; FDI = first dorsal interosseous; APB = abductor pollicis brevis; RMT = resting motor threshold; AMT = active motor threshold. 
A

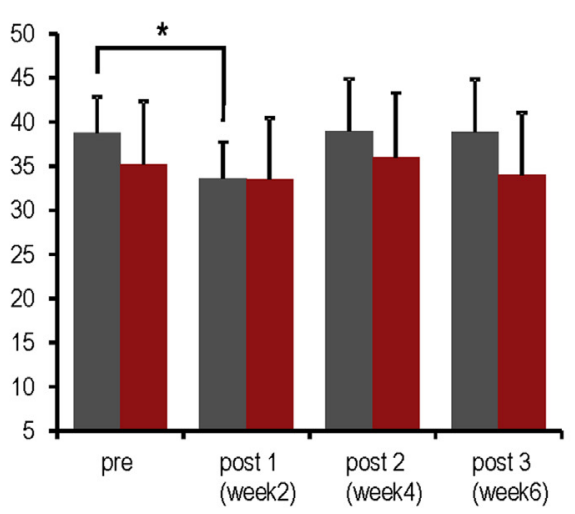

B

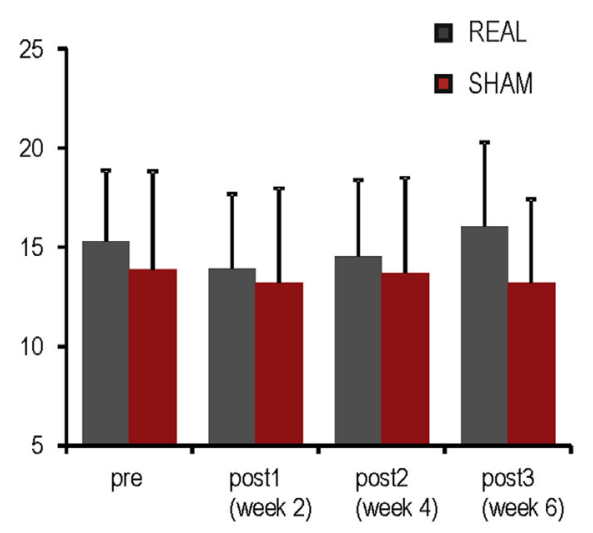

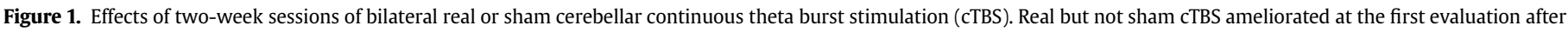
two weeks of treatment TWRST scores (A) but not the BFMDRS scores (B). ${ }^{*} P<0.05$. Error bars indicate SEM.

surround inhibition [5]. As reported previously we found that patients with $\mathrm{CD}$ in comparison with HS had an increased response to the median nerve PAS protocol, with enhanced facilitation of MEPs in both the median innervated APB muscle as well as the ulnar innervated FDI $[5,44]$ as revealed by ANOVA showing that there were no effects for the GROUP $(F(2,25)=1.52 ; P=0.23)$ and $\operatorname{MUSCLE}(F(1,25)=0.34 ; P=0.56)$ main factors, while there was a significant GROUP $\times$ MUSCLE interaction: $(F(2,25)=3.95$; $P=0.030$ ). Post-hoc analysis showed that healthy controls in comparison with $C D$ patients had no facilitation for the FDI muscle $(P<0.05$ in comparison with both the cTBS and the sham groups) (Fig. 2D).

In the cTBS group two weeks of cerebellar stimulation modified the CBI circuits over contralateral M1. There was a significant
ISI $(F(2,32)=3.26 ; P=0.04)$, but not $\operatorname{TIME}(F(1,16)=2.46$; $P=0.62)$ main factor and a significant GROUP $\times$ TIME $\times$ ISI interaction $(F(2,32)=4.28 ; P=0.02)$. Post hoc analysis showed that changes occurred after real and not sham cerebellar cTBS at ISI $=10 \mathrm{~ms}(P=0.03)$ in which CBI was reduced (Fig. 3$)$. Cerebellar stimulation did not modify SICI/ICF data in the two CD groups. There was only a significant effect for the ISI main factor $(F(6,96)=12.44 ; P=0.00001)$ but not for the GROUP $(F(1,16)=0.87 ; P=0.36)$ nor TIME $(F(1,16)=1.44 ; P=0.24)$ main factors, although in the CTBS group SICI values tended to be reduced across all ISIs. However, this effect did not reach statistical evidence (TIMEx ISI interaction $(F=1.28 ; P=0.24)$ ) (Fig. 4). CSP was not modified by the two weeks of cerebellar cTBS as revealed by ANOVA failing to show any effect for both the GROUP

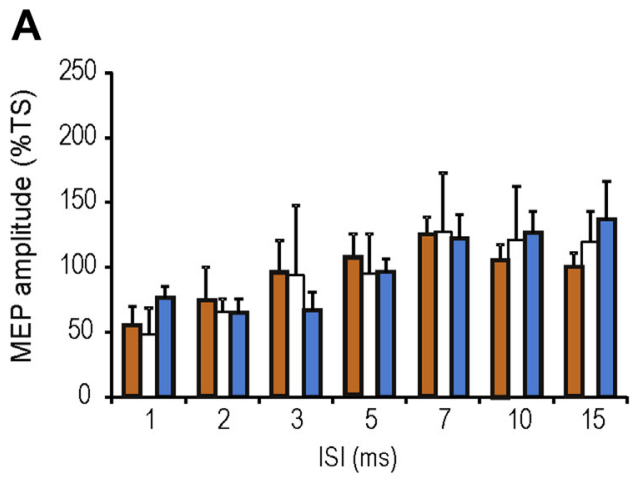

C

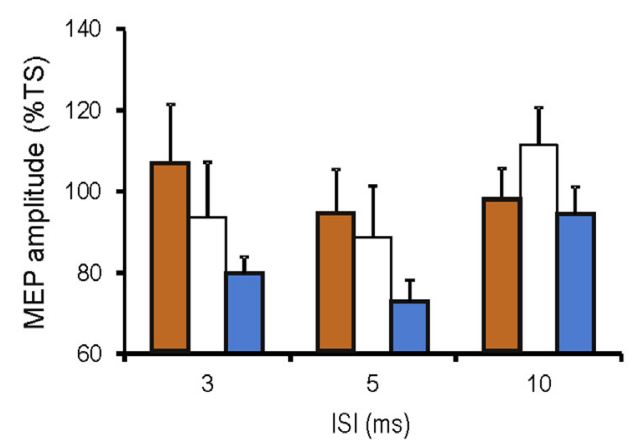

B

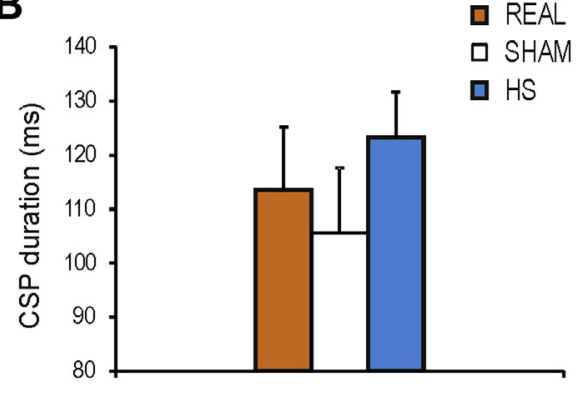

D

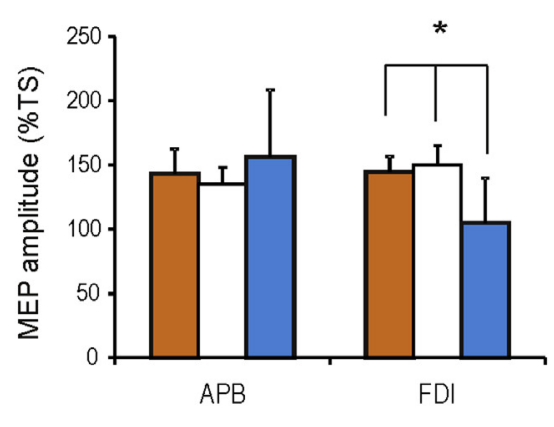

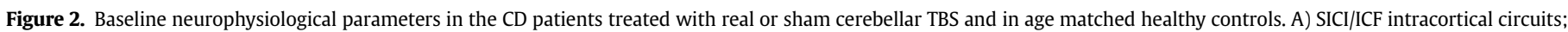
B) cortical silent period; C) cerebellar brain inhibition; D) paired associative stimulation. 

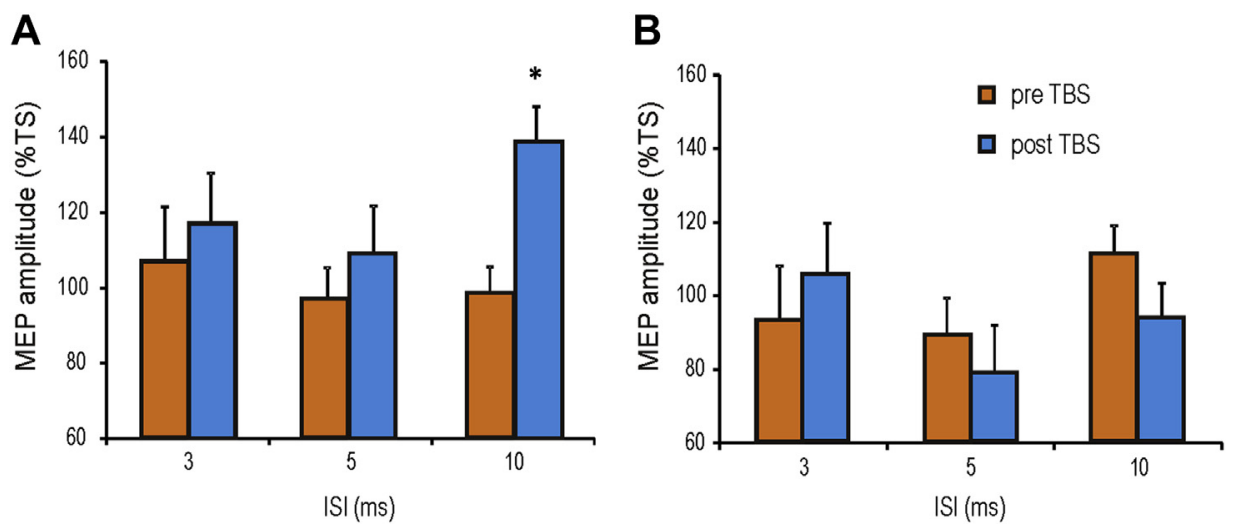

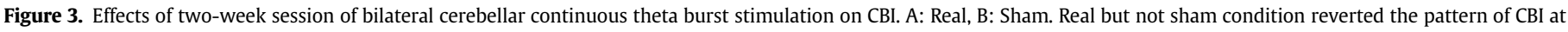
$10 \mathrm{~ms}$, leading to a facilitation of M1. ${ }^{*} P<0.05$. Error bars indicate SEM.

$(F(1,16)=0.007 ; P=0.94)$ nor $\operatorname{TIME}(F(1,16)=1.62 ; P=0.22)$ main factors and no interaction (Fig. 5).

After two weeks of cerebellar stimulation we found for PAS that in the cTBS group the potentiation on the ulnar innervated FDI disappeared, leading to a normal pattern of topographic specific induced plasticity. On the other hand this was not the case in the sham group, in which the same altered pattern was observed after two weeks of stimulation. ANOVA performed on PAS data revealed no significant GROUP $(F(1,16)=0.14 ; P=0.71)$, MUSCLE $(F(1,16)=0.16 ; P=0.79)$ and $\operatorname{TIME}(F(1,16)=2.74 ; P=0.11)$ main factors, but significant MUSCLE $\times \operatorname{TIME}(F(1,16)=4.38 ; P=0.05)$ and MUSCLE $\times$ TIME $\times$ GROUP interactions $(F(1,16)=4.12$; $P=0.04)$. Post hoc analysis showed that only the FDI muscle differed in the cTBS group before and after PAS $(P<0.05)$ (Fig. 6). The correlation analysis performed between the percentage of change of PAS and the percentage of change in the TWTRS scores after two weeks of real stimulation did not show any significant effect.

\section{Discussion}

Our findings demonstrate that in patients with dystonia the long-lasting modulation of CTC circuits induces some clinical and neurophysiological effects. These data provide novel evidence that non-invasive stimulation of the cerebellum could be a potential way to control some symptoms and to modulate altered mechanisms of cortical plasticity in CD patients.

Notably, the current study represents one of the first attempts in which non-invasive brain stimulation was applied during two weeks in a controlled design to test the clinical efficacy of TMS in patients with CD. Previous studies reported behavioral improvements in patients with focal hand dystonia, especially when rTMS was applied over the premotor cortex, but most were single-session proof of principle studies with small samples of patients [45-47]. The analysis of clinical scores resulting from our dual-center double-blind randomized controlled showed that cerebellar cTBS induced a significant improvement that was evident on the first evaluation after the end of the two weeks of cerebellar stimulation, but disappeared in the following evaluations. In contrast with the long lasting beneficial effect induced by cerebellar cTBS in PD patients with levodopa-induced dyskinesias (LIDs) [32,33], here we found that the clinical impact on dystonic symptoms was more transient. This difference could be ascribed to the same mechanisms underlying a late clinical response to deep brain stimulation that characterizes patients with dystonia; while in Parkinson's disease the maximal clinical response occurs within hours of switching on the device, it can take weeks or months to achieve maximal clinical benefit in dystonia $[48,49]$. Therefore an open question is whether more prolonged sessions of cerebellar cTBS could be effective in inducing more profound and sustained clinical changes. In alternative it is possible that different clinical result could also emerge when applying over the cerebellum other protocols of TMS, such as $1 \mathrm{~Hz}$ rTMS or intermittent TBS $[27,40]$, or other methods of non-invasive stimulation such as transcranial direct current stimulation (TDCS) $[50,51]$.

Despite these considerations, it is likely that the clinical beneficial effects observed here could involve a direct inhibitory effect of cTBS on an abnormal enhanced activity of the cerebellar cortex [13]. It has been noticed that the types of lesions affecting the cerebellum and causing dystonia more commonly cause a distortion or increase
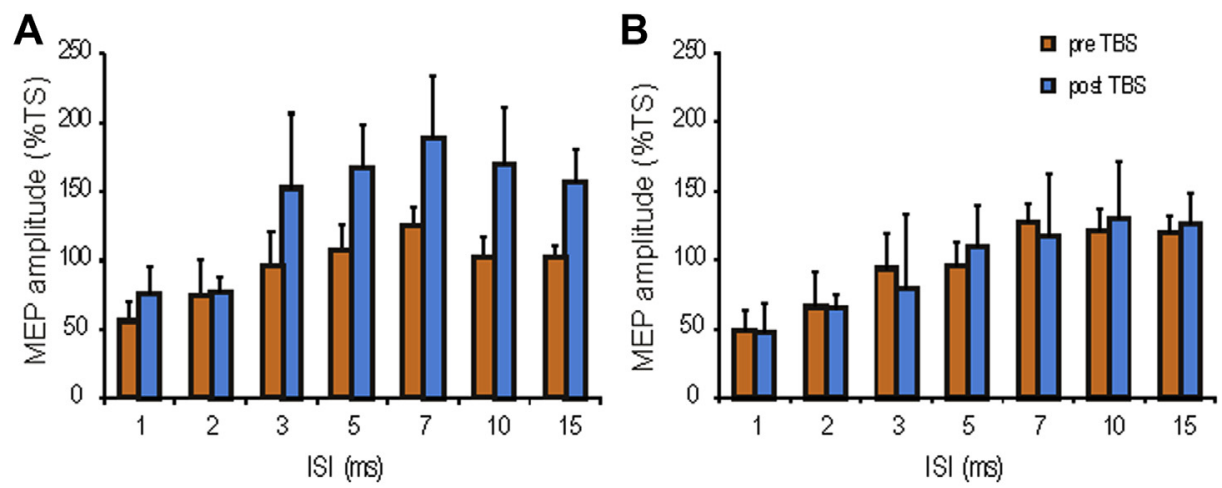

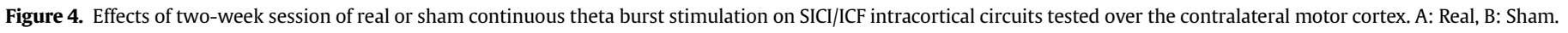




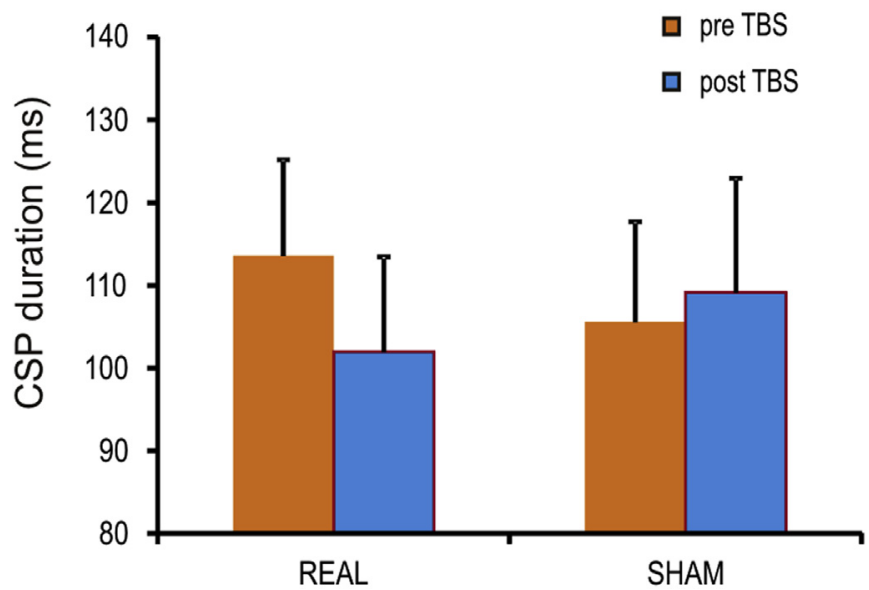

Figure 5. Effects of two-weeks of bilateral real or sham cerebellar continuous theta burst stimulation on the CSP tested over the contralateral motor cortex.

in Purkinje neuron activity (i.e. posterior fossa tumors) rather than a loss of activity (as in stroke). Interestingly, several years ago it was proposed that there is a key difference between "irritative" and "paralytic" cerebellar lesions in relation to cervical dystonia [13]. Accordingly, experimental evidence in animal models of dystonia demonstrated that cerebellar activity is increased in relation with the occurrence of dystonic postures [16,52]. Indeed, in animal models the surgical removal of the cerebellum eliminated the dystonic attacks, suggesting the cerebellum was the source of the problem [15].

Clinical changes were paralleled by relevant neurophysiological modifications. We found that CBI was modulated by cerebellar cTBS. This finding is in agreement with previous observations in healthy controls [28]. It has been proposed that cerebellar cTBS changes these $\mathrm{CBI}$ interactions trough a local effect on the Purkinje cells [28]. Indeed, using PET we recently observed that cerebellar cTBS causes a decrease in local metabolism that is confined to the cerebellum [53]. Therefore, it is likely that the observed effects could involve a direct modulation of Purkinje cells. However, there is no conclusive evidence that $\mathrm{CBI}$ interactions could be directly related to other mechanisms of cortical plasticity or other interconnected inhibitory circuits. For instance, other investigations in healthy controls showed that bidirectional change of $\mathrm{CBI}$ after anodal (CBI increase) vs. cathodal TDCS (CBI decrease) of the lateral cerebellum [51], or a reduction of CBI after $1 \mathrm{~Hz}$ rTMS or continuous theta-burst stimulation [28] can be seen without changes in MEP amplitude, suggesting hat the modifications of M1 excitability (indexed by MEP amplitude) and CBI are often dissociated [54]. Moreover we observed a reversal of the CBI toward facilitation at ISI $=10 \mathrm{~ms}$. This result was rather unexpected, since the most prominent inhibitory interaction is usually observed at shorter ISIs of $5-7 \mathrm{~ms}[21,22]$. It is possible that the interaction at ISI $=10 \mathrm{~ms}$ could involve polysynaptic circuits involving an additional relay in the premotor areas, that are markedly modulated by the cerebellar projections [24]. It has to be noticed that the CBI interactions at ISI $=10 \mathrm{~ms}$ have not been sufficiently investigated in previous studies, especially in healthy controls, although some abnormalities have been observed in patients with parkinsonism [55]. Therefore, we cannot exclude that the effect reported in our study could involve the activation of some other structures adjacent to the cerebellum, such as the neck muscles.

Two weeks of cerebellar stimulation in the cTBS group abolished the potentiation on the ulnar innervated FDI, leading to a normal pattern of topographic specific induced plasticity. Several studies have tested the PAS protocols in focal dystonic patients, reporting reliable and consisting findings $[5,6,43,56]$. First, associative plasticity after PAS is increased; more importantly there is a profound loss of spatial specificity so that the facilitation can be seen in both median and ulnar innervated muscles and not only in the target muscle as in healthy controls $[5,43]$. Such loss of spatial specificity is considered one of the most important and robust finding. Its pathophysiology has been put in direct relation with the abnormalities of neuronal inhibition identified previously both in the motor and somatosensory system in dystonic patients [57], in the context of a failure of neuronal inhibition. Remarkably, this excess of motor cortex plasticity is not restricted to the circuits clinically affected by dystonia but generalize across the entire sensorimotor system, suggesting that it may represent an endophenotypic trait of the disease $[6,58]$.

Cerebellar CTBS was effective in counteracting the loss of spatial specificity observed in our sample of CD patients. This strong interplay among cerebellar TBS and cortical plasticity was not unexpected, given that recent evidence showed that the lateral cerebellum controls some cortical plastic mechanisms. For instance Hamada et al. [50] showed that simultaneous TDCS (2 mA) over the cerebellum can abolish the PAS effect entirely. Surprisingly, the effect is seen when the PAS interval is $25 \mathrm{~ms}$ but not when it is $21.5 \mathrm{~ms}$, suggesting that these protocols involve different neural circuits, one under the control of the cerebellar projections and the other by-passing the cerebellum. Similarly, Popa et al. [31] found that in healthy controls the plasticity induced in M1 by PAS was enhanced by inhibitory stimulation of cerebellar cortex. Such a plasticity-enhancing effect of cerebellar stimulation was not seen when TBS, which does not involve peripheral sensory input, was
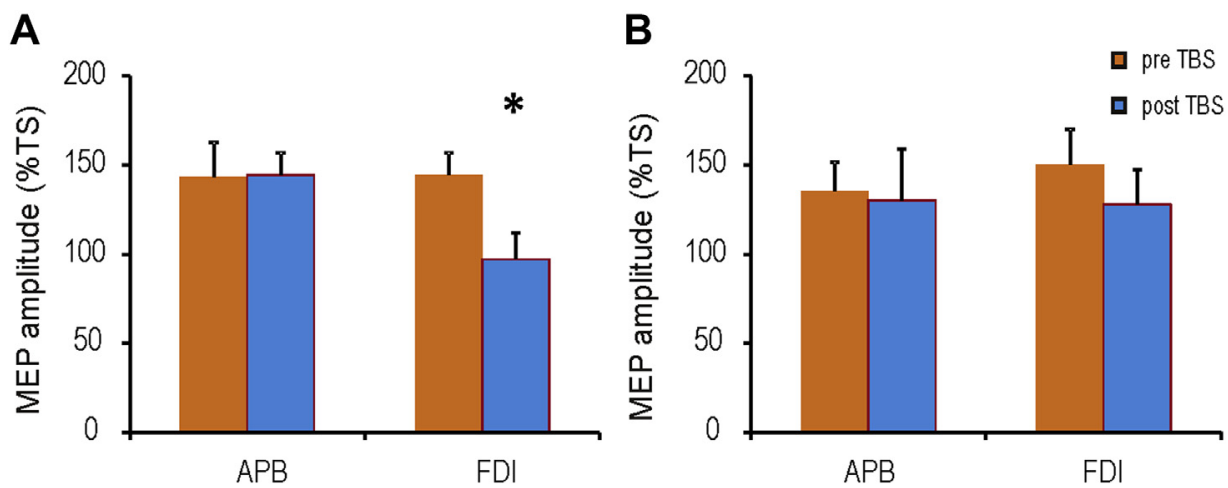

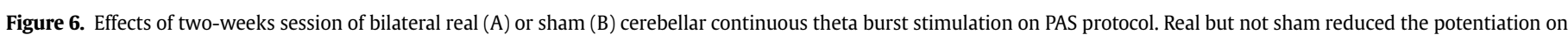
the ulnar innervated FDI. 
used instead of PAS to stimulate M1. Taken together, these findings provided strong evidence that the cerebellum or interconnected structures, such as the thalamus or the dentato-olivary nuclear complex could have an important role in controlling the plastic changes occurring within the cortex, essentially by modulating the sensory afferents upstream of M1.

Notably, cerebellar cTBS has also been reported to reduce levo-dopa induced dyskinesias in PD patients [32,33], providing evidence for a dysfunctional interplay between the striato-thalamo-cortical and the cerebello-thalamo-cortical networks.

In particular it has been proposed that abnormal signaling in the basal ganglia circuits could result in some alterations in the sensory processing function of the cerebellum. This would lead to an inappropriate filtering of the relevant sensory volley, which could be responsible for a maladaptive state of cortical plasticity and thus generate abnormal and unwanted motor programs [33]. Within this view it is plausible that in focal dystonic patients cerebellar stimulation, by increasing the gain of the sensory afferent volley to M1, could permit a better sensorimotor integration [59].

The long lasting modulation of the CTC pathways did not change local inhibitory activity in M1. This is in contrast with previous studies showing that especially GABA(B) dependent inhibitory interneurons in M1 are modulated by cerebellar TBS [27]. In healthy controls cerebellar cTBS is supposed to increases the activation of presynaptic GABA(B) receptors as showed by a decrease of SICI, while increases $\operatorname{GABA}(B)$ receptor mediated inhibitory post-synaptic potentials as revealed by changes in long intracortical inhibition (LICI) [27,60]. This explanation is also supported in other studies reporting that dystonic patients have shortened cortical silent period (CSP) [43,61], implying a decreased efficiency of GABA(B) inhibitory activity in M1 [60]. In the current study, cerebellar cTBS failed to modulate these circuits in our sample of dystonic patients, although there was a trend in the same direction as previously described in healthy controls. This could suggest that the cerebellum controls the mechanisms of cortical plasticity and of cortical inhibition trough different interactions. A limitation of the current study is that the electrophysiological effects of cTBS were only assessed in patients. It would have been relevant to contrast the effects in dystonia to those in age matched healthy controls.

The current data could be taken as a novel evidence in favor of a "network model" at the basis of dystonia, extending the view that consider the basal ganglia dysfunction as the sole cause $[7,10,13]$. This model accommodates the possibility that dystonia may result from dysfunction of other nodes in the network, from combined dysfunction of multiple nodes, or from aberrant communication among the nodes. Notably, a strong anatomofunctional interplay among the basal ganglia and the cerebellum has been recently described in primates [62]. An anatomical substrate exists for cerebellar output to influence the input stage of the basal ganglia and vice-versa. These interconnections provide the neural basis for cerebellar involvement in what have typically been considered to be basal ganglia operations, such as reward-related learning, and in so-called basal ganglia disorders, such as Parkinson's disease, dystonia, or Tourette syndrome [63].

In conclusion our study provide novel physiological evidence that in dystonia the cerebellum could be a potential target to control some altered mechanisms of cortical plasticity. Further studies are needed to evaluate the impact of cerebellar cTBS in other forms of generalized or inherited dystonia and to better clarify the interaction between the CTC pathways and the basal ganglia circuits.

\section{References}

[1] Hallett M. Dystonia: abnormal movements result from loss of inhibition. Adv Neurol 2004:94:1-9.

[2] Byl NN, Merzenich MM, Cheung S, Bedenbaugh P, Nagarajan SS, Jenkins WM. A primate model for studying focal dystonia and repetitive strain injury: effects on the primary somatosensory cortex. Phys Ther 1997;77(3):269-84.

[3] Martella G, Tassone A, Sciamanna G, et al. Impairment of bidirectional synaptic plasticity in the striatum of a mouse model of DYT1 dystonia: role of endogenous acetylcholine. Brain 2009;132(Pt 9):2336-49.

[4] Stefan K, Kunesch E, Cohen LG, Benecke R, Classen J. Induction of plasticity in the human motor cortex by paired associative stimulation. Brain 2000; 123(Pt 3):572-84.

[5] Quartarone A, Bagnato S, Rizzo V, et al. Abnormal associative plasticity of the human motor cortex in writer's cramp. Brain 2003;126(Pt 12):2586-96.

[6] Quartarone A, Morgante F, Sant'angelo A, et al. Abnormal plasticity of sensorimotor circuits extends beyond the affected body part in focal dystonia. J Neurol Neurosurg Psychiatry 2008;79(9):985-90.

[7] Jinnah HA, Hess EJ. A new twist on the anatomy of dystonia: the basal ganglia and the cerebellum? Neurology 2006;67(10):1740-1.

[8] Calderon DP, Fremont R, Kraenzlin F, Khodakhah K. The neural substrates of rapid-onset Dystonia-Parkinsonism. Nat Neurosci 2011;14(3):357-65.

[9] Delmaire C, Vidailhet M, Elbaz A, et al. Structural abnormalities in the cerebellum and sensorimotor circuit in writer's cramp. Neurology 2007;69(4): 376-80.

[10] Carbon M, Eidelberg D. Abnormal structure-function relationships in hereditary dystonia. Neuroscience 2009;24(1):164.

[11] Carbon M, Argyelan M, Eidelberg D. Functional imaging in hereditary dystonia. Eur J Neurol 2010;17(Suppl. 1):58-64.

[12] Argyelan M, Carbon M, Niethammer M, et al. Cerebello-thalamo-cortical connectivity regulates penetrance in dystonia. J Neurosci 2009;29(31):9740-7.

[13] Neychev VK, Gross RE, Lehéricy S, Hess EJ, Jinnah HA. The functional neuroanatomy of dystonia. Neurobiol Dis 2011;42(2):185-201.

[14] Zadro I, Brinar VV, Barun B, Ozretić D, Habek M. Cervical dystonia due to cerebellar stroke. Mov Disord 2008;23(6):919-20.

[15] Neychev VK, Fan X, Mitev VI, Hess EJ, Jinnah HA. The basal ganglia and cerebellum interact in the expression of dystonic movement. Brain 2008; 131(Pt 9):2499-509.

[16] Chen G, Popa LS, Wang X, et al. Low-frequency oscillations in the cerebellar cortex of the tottering mouse. J Neurophysiol 2009;101:234-45.

[17] Prudente CN, Pardo CA, Xiao J, et al. Neuropathology of cervical dystonia. Exp Neurol 2013;241:95-104.

[18] Hubsch C, Roze E, Popa T, et al. Defective cerebellar control of cortical plasticity in writer's cramp. Brain 2013;136:2050-62.

[19] Middleton FA, Strick PL. Basal ganglia output and cognition: evidence from anatomical, behavioral, and clinical studies. Brain Cogn 2000;42(2): 183-200.

[20] Dum RP, Strick PL. An unfolded map of the cerebellar dentate nucleus and its projections to the cerebral cortex. J Neurophysiol 2003;89(1):634-9.

[21] Ugawa Y, Uesaka Y, Terao Y, Hanajima R, Kanazawa I. Magnetic stimulation over the cerebellum in humans. Ann Neurol 1995 Jun;37(6):703-13.

[22] Pinto AD, Chen R. Suppression of the motor cortex by magnetic stimulation of the cerebellum. Exp Brain Res 2001;140(4):505-10.

[23] Daskalakis ZJ, Paradiso GO, Christensen BK, Fitzgerald PB, Gunraj C, Chen R. Exploring the connectivity between the cerebellum and motor cortex in humans. J Physiol 2004;557(Pt 2):689-700.

[24] Ito M. Cerebellar long-term depression: characterization, signal transduction, and functional roles. Physiol Rev 2001;81(3):1143-95.

[25] D'Angelo E, Rossi P, Armano S, Taglietti V. Evidence for NMDA and mGlu receptor-dependent long-term potentiation of mossy fiber-granule cell transmission in rat cerebellum. J Neurophysiol 1999;81(1):277-87.

[26] Jörntell H, Hansel C. Synaptic memories upside down: bidirectional plasticity at cerebellar parallel fiber-Purkinje cell synapses. Neuron 2006; 52(2):227-38.

[27] Koch G, Mori F, Marconi B, et al. Changes in intracortical circuits of the human motor cortex following theta burst stimulation of the lateral cerebellum. Clin Neurophysiol 2008;119(11):2559-69.

[28] Popa T, Russo M, Meunier S. Long-lasting inhibition of cerebellar output. Brain Stimul 2010;3(3):161-9.

[29] Huang YZ, Edwards MJ, Rounis E, Bhatia KP, Rothwell JC. Theta burst stimulation of the human motor cortex. Neuron 2005;45(2):201-6.

[30] Koch G. Repetitive transcranial magnetic stimulation: a tool for human cerebellar plasticity. Funct Neurol 2010;25(3):159-63.

[31] Popa T, Velayudhan B, Hubsch C, et al. Cerebellar processing of sensory inputs primes motor cortex plasticity. Cereb Cortex 2013 Feb;23(2):305-14.

[32] Koch G, Brusa L, Carrillo F, et al. Cerebellar magnetic stimulation decreases levodopa-induced dyskinesias in Parkinson disease. Neurology 2009;73(2): 113-9.

[33] Kishore A, Popa T, Balachandran A, et al. Cerebellar sensory processing alterations impact motor cortical plasticity in Parkinson's disease: clues from dyskinetic patients. Cereb Cortex; 2013 Mar 27 [Epub ahead of print].

[34] Consky E, Basinkmi A, Bellem L, Ranawayam R, Lang A. The Toronto Western Torticollis Rating Scale (TWSTRS): assessment of validity and inter-rater reliability. Neurology 1990;40:445. 
[35] Burke RE, Fahn S, Marsden CD, Bressman SB, Moskowitz C, Friedman J. Validity and reliability of a rating scale for the primary torsion dystonias. Neurology 1985;35(1):73-7.

[36] Rossini PM, Barker AT, Berardelli A, et al. Non-invasive electrical and magnetic stimulation of the brain, spinal cord and roots: basic principles and procedures for routine clinical application. Report of an IFCN committee. Electroencephalogr Clin Neurophysiol 1994;91(2):79-92.

[37] Rothwell JC. Techniques and mechanisms of action of transcranial stimulation of the human motor cortex. J Neurosci Methods 1997;74(2):113-22.

[38] Kujirai T, Caramia MD, Rothwell JC, et al. Cortico-cortical inhibition in human motor cortex. J Physiol 1993;471:501-19.

[39] Del Olmo MF, Cheeran B, Koch G, Rothwell JC. Role of the cerebellum in externally paced rhythmic finger movements. J Neurophysiol 2007; 98(1):145-52.

[40] Oliveri M, Koch G, Torriero S, Caltagirone C. Increased facilitation of the primary motor cortex following $1 \mathrm{~Hz}$ repetitive transcranial magnetic stimulation of the contralateral cerebellum in normal humans. Neurosci Lett 2005; 376(3):188-93.

[41] Carrillo F, Palomar FJ, Conde V, et al. Study of cerebello-thalamocortical pathway by transcranial magnetic stimulation in Parkinson's disease. Brain Stimul 2013;6(4):582-9.

[42] Cantello R, Gianelli M, Civardi C, Mutani R. Magnetic brain stimulation: the silent period after the motor evoked potential. Neurology 1992;42(10): 1951-9.

[43] Kang JS, Terranova C, Hilker R, Quartarone A, Ziemann U. Deficient homeostatic regulation of practice-dependent plasticity in writer's cramp. Cereb Cortex 2011;21(5):1203-12.

[44] Weise D, Schramm A, Stefan K, et al. The two sides of associative plasticity in writer's cramp. Brain 2006;129(Pt 10):2709-21.

[45] Murase N, Rothwell JC, Kaji R, et al. Subthreshold low-frequency repetitive transcranial magnetic stimulation over the premotor cortex modulates writer's cramp. Brain 2005;128:104-15.

[46] Siebner HR, Filipovic SR, Rowe JB, et al. Patients with focal arm dystonia have increased sensitivity to slow-frequency repetitive TMS of the dorsal premotor cortex. Brain 2003;126:2710-25.

[47] Lefaucheur JP, Fénelon G, Ménard-Lefaucheur I, Wendling S, Nguyen JP. Lowfrequency repetitive TMS of premotor cortex can reduce painful axial spasms in generalized secondary dystonia: a pilot study of three patients. Clin Neurophysiol 2004;34:141-5.
[48] Hammond C, Ammari R, Bioulac B, Garcia L. Latest view on the mechanism of action of deep brain stimulation. Mov Disord 2008;23(15):2111-21.

[49] Sensi M, Cavallo MA, Quatrale R, et al. Pallidal stimulation for segmental dystonia: long term follow up of 11 consecutive patients. Mov Disord 2009;24(12):1829-35.

[50] Hamada M, Strigaro G, Murase N, et al. Cerebellar modulation of human associative plasticity. J Physiol 2012;590:2365-74.

[51] Galea JM, Jayaram G, Ajagbe L, Celnik P. Modulation of cerebellar excitability by polarity-specific noninvasive direct current stimulation. J Neurosci 2009 Jul 15;29(28):9115-22.

[52] Pizoli CE, Jinnah HA, Billingsley ML, Hess EJ. Abnormal cerebellar signaling induces dystonia in mice. J Neurosci 2002;22(17):7825-33.

[53] Brusa L, Ceravolo R, Kiferle L, et al. Metabolic changes induced by theta burst stimulation of the cerebellum in dyskinetic Parkinson's disease patients. Parkinsonism Relat Disord 2012;18(1):59-62.

[54] Lu MK, Tsai CH, Ziemann U. Cerebellum to motor cortex paired associative stimulation induces bidirectional STDP-like plasticity in human motor cortex. Front Hum Neurosci 2012;6:260.

[55] Brusa L, Ponzo V, Mastropasqua C, et al. Theta burst stimulation modulates cerebellar-cortical connectivity in patients with progressive supranuclear palsy. Brain Stimul 2014 Jan-Feb;7(1):29-35.

[56] Belvisi D, Suppa A, Marsili L, et al. Abnormal experimentally- and behaviorally-induced LTP-like plasticity in focal hand dystonia. Exp Neurol 2013;240:64-74.

[57] Hallett M, Weiner WJ, Kompoliti K. Psychogenic movement disorders. Parkinsonism Relat Disord 2012 Jan;18(Suppl. 1):S155-7.

[58] Quartarone A, Pisani A. Abnormal plasticity in dystonia: disruption of synaptic homeostasis. Neurobiol Dis 2011;42(2):162-70.

[59] Tinazzi M, Fiorio M, Fiaschi A, Rothwell JC, Bhatia KP. Sensory functions in dystonia: insights from behavioral studies. Mov Disord 2009;24(10):1427-36.

[60] McDonnell MN, Ridding MC. Transient motor evoked potential suppression following a complex sensorimotor task. Clin Neurophysiol 2006;117(6): 1266-72.

[61] Filipović SR, Ljubisavljević M, Svetel M, Milanović S, Kacar A, Kostić VS. Neurosci Lett 1997;222(3):167-70.

[62] Bostan AC, Dum RP, Strick PL. The basal ganglia communicate with the cerebellum. Proc Natl Acad Sci U S A 2010;107(18):8452-6.

[63] Bostan AC, Dum RP, Strick PL. Cerebellar networks with the cerebral cortex and basal ganglia. Trends Cogn Sci 2013;17(5):241-54. 